\title{
MicroRNA-126 inhibits proliferation and metastasis by targeting pik3r 2 in prostate cancer
}

\author{
LEI SONG, XUBIO XIE, SHAOJIE YU, FENGHUA PENG and LONGKAI PENG \\ Department of Urological Organ Transplantation, The Second Xiangya Hospital of Central South University, \\ Changsha, Hunan 410011, P.R. China
}

Received January 11, 2015; Accepted November 10, 2015

DOI: $10.3892 / \mathrm{mmr} .2015 .4661$

\begin{abstract}
The dysregulation of miR-126 has been reported to correlate with the progression of several cancer types. The present study demonstrated that miR-126 was significantly downregulated in prostate cancer (PCa) tissues compared with normal prostate tissues. In vitro and in vivo studies indicated that forced overexpression of miR-126 significantly suppressed the proliferation of PCa cell lines. Additionally, a Transwell assay showed that enhanced expression of miR-126 inhibited metastasis in $\mathrm{PCa}$ in vitro. Furthermore, pik3r2 was confirmed to be a direct target of miR-126 in PCa. It was also shown that pik3r2 was upregulated in PCa tissues and this inversely correlated with miR-126 in PCa tissues. In conclusion, these results revealed that aberrant expression of miR-126 promoted the progression of $\mathrm{PCa}$ and may serve as a novel therapeutic biomarker for $\mathrm{PCa}$.
\end{abstract}

\section{Introduction}

Prostate cancer ( $\mathrm{PCa}$ ) is one of the most frequently diagnosed and mortality-associated cancer types in men, particularly in western countries (1). The morbidity and mortality of PCa are markedly increasing in China in previous years (2). Although patients with localized $\mathrm{PCa}$ are reported to have a survival rate of $>5$ years $(3,4)$, it is hypothesized that few effective therapeutic options are effective for advanced $\mathrm{PCa}$, even if applying advanced therapeutic strategies. A previous study demonstrated that in the USA, there were an estimated 240,000 new cases and 33,000 deaths in 2011, predominantly due to its high potential for tumor progression (5). Molecular strategies for the diagnosis and treatment of cancer have been developing (6,7). Therefore, the detection of efficient diagnostic and prognostic biomarkers for $\mathrm{PCa}$ is becoming extremely

Correspondence to: Mr. Longkai Peng, Department of Urological Organ Transplantation, The Second Xiangya Hospital of Central South University, 139 Middle Renming Road, Changsha, Hunan 410011, P.R. China

E-mail: plk3329@126.com

Key words: prostate cancer, miR-126, pik3r2, proliferation, metastasis necessary for the improvement of the clinical outcome of patients with this disease. Micro (mi)RNAs are a class of short (19-22 nucleotides), non-coding RNA sequences, which negatively regulate their target genes post-transcriptionally (8). There are two ways in which miRNAs regulate their target genes, by either promoting mRNA degradation or repressing protein translation through sequence-specific interaction with the 3'-untranslated region of targeted mRNA $(9,10)$. Previous studies have shown that deregulation of miRNAs is closely associated with the process of tumor progression, including inhibiting apoptosis (11), promoting proliferation (12), invasion (13) and angiogenesis (14). In PCa, it is reported that deregulation of miRNAs are vital in the initiation and progression of PCa, including miR-361-5p (15) and miR-26a (16). To date, miR-126 has been demonstrated to be downregulated in several cancer types, including cervical (17) and colon (18). A previous study revealed that the expression level of miR-126 is significantly decreased in PCa tissues and the expression of miR-126 is an independent prognostic factor of PCa following radical prostatectomy (19). In the present study, the underlying mechanism of miR-126 in mediating PCa progression was the primary focus. In vitro investigations showed that miR-126 markedly suppressed the ability of PCa cells to invade extracellular matrix gel and proliferate. Furthermore, the data indicated that pik3r2 was a direct target of miR-126 in PCa cells. In clinical tissues, it was also confirmed that the expression of miR-126 and pik3r2 were inversely correlated. Additionally, in vivo experiments revealed that miR-126 had an obvious antiproliferative effect. These findings highlighted the important functions of miR-126 in PCa initiation and progression, and therefore, miR-126 may be a promising therapeutic molecular target for $\mathrm{PCa}$.

\section{Materials and methods}

Patients and samples. PCa tissues enrolled in the present study were obtained from 20 patients who underwent radical prostatectomy and had not received any previous treatment in our center (The Second Xiangya Hospital of Central South University, Changsha, China). For frozen specimens, surgical pathologists examined the clinicopathological features of freshly frozen sections following staining with hematoxylin and eosin and subsequently the samples were snap frozen in liquid nitrogen. Only those samples with $>70 \%$ tumor content 
were included in the present study. The present study was approved by the Ethics Committee of The Second Xiangya Hospital of Central South University (no. S048, 2013) and were performed after obtaining written informed consent from each of the patients.

$R N A$ preparation and reverse transcription-quantitative polymerase chain reaction ( $R T-q P C R)$. The total RNA was extracted from tissues or cells using TRIzol reagent (Invitrogen; Thermo Fisher Scientific, Inc., Waltham, MA, USA), according to the manufacturer's protocol. A total of $2 \mu \mathrm{g}$ RNA was transcribed into cDNA using MMLV reverse transcriptase (Invitrogen; Thermo Fisher Scientific, Inc.) for mRNA and an RT-PCR kit (RR716, Takara Bio Inc., Otsu, Japan) for miRNA. All qPCR reactions were performed with an ABI7500fast system (Applied Biosystems; Thermo Fisher Scientific, Inc.). The primers for miR-126 and RNU6B were purchased from Takara Bio Inc. The sequences of the other primers were as follows: Pik3r2, forward: 5'-ATGGCACCTTCCTAGTCCGAGA-3' and reverse: 5'-CTCTGAGAAGCCATAGTGCCCA-3'; GAPDH, forward: 5'-GTCTCCTCTGACTTCAACAGCG-3' and reverse: 5'-ACC ACCCTGTTGCTGTAGCCAA-3'; and TGCGGGTGCTCG TTCGGCAGC and the Universal Primer (Takara Bio, Inc.) for RNU6B. GAPDH and RNU6B were used as internal controls.

Cell culture. Human DU145, PC-3 and 293T PCa cell lines were purchased from the Shanghai Institute of Cell Biology (Shanghai, China). The DU-145 and PC-3 cells were cultured in RPMI-1640 medium (Hyclone, Logan, UT, USA) and the $293 \mathrm{~T}$ cells were cultured in Dulbecco's modified Eagle's medium (Hyclone). Each culture medium was supplemented with $10 \%$ fetal bovine serum (FBS; Gibco; Thermo Fisher Scientific, Inc.) and the cells were maintained at $37^{\circ} \mathrm{C}$ in a humidified environment, containing $5 \% \mathrm{CO}_{2}$.

Oligonucleotides and cell transfection. miR-126 mimics and its negative control (NC) were designed and chemically synthesized by GenePharma (Shanghai, China). For transfection, the cells were plated into 6 -well plates with $2.5 \times 10^{4}$ cells/well. Once the cells were $30-50 \%$ confluent, miR-126 mimics or NC, accompanied with Lipofectamine 2000 (Invitrogen; Thermo Fisher Scientific, Inc.) were transfected into the PCa cells. The medium was changed following a $24 \mathrm{~h}$ incubation.

Cell proliferation assay. The DU145 and PC-3 cells were seeded and transfected in 6-well plates with $2.5 \times 10^{4}$ cells/well, and were subsequently transferred into 96-well plates with 3,000 cells/well $24 \mathrm{~h}$ following the transfection. The proliferation of the cells was detected using a Cell Counting kit-8 reagent (Dojindo Laboratories, Kyushu Island, Japan), according to the manufacturer's protocol. The relative numbers of viable cells were represented by the absorbance at a wavelength of $450 \mathrm{~nm}$ following 24, 48 and $72 \mathrm{~h}$ incubation in 96-well plates (UV-6100; Shanghai Mapada Instruments, Co., Ltd., Shanghai, China). The experiments were performed in triplicate.

Migration and invasion assays of PCa cells. The migration and invasion of PCa cells were analyzed in 24-well Boyden chambers with $8 \mu \mathrm{m}$ pore size polycarbonate membranes (Corning,
Inc., Corning, NY, USA). A total of $4 \times 10^{4}$ transfected cells were suspended in the upper chamber with serum-free medium, while the lower chamber was filled with $10 \%$ FBS-containing medium. In the invasion assays, the membranes were covered with Matrigel (BD Biosciences, San Diego, CA, USA) to form matrix barriers. Following incubation for 30 (migration assay) or $48 \mathrm{~h}$ (invasion assay), the cells on the upper surface were removed by wiping with a cotton swab and the cells on the lower surface of the membrane were fixed in methanol, stained with $0.1 \%$ crystal violet (Sigma-Aldrich, St. Louis, MO, USA) and counted. The experiments were repeated independently three times.

Plasmid construction and luciferase reporter assay. The binding site of miR-126 and pik3r2 was predicted by TargetScan (www.targetscan.org) and miRanda (www.microrna.org). To form a wild-type luciferase reporter plasmid of pik3r2 (Guangzhou RiboBio Co., Ltd., Guangzhou, China), the binding segment was chemosynthesized, amplified and cloned into the firefly luciferase reporter plasmid. The binding site was subsequently altered to form a mutant type luciferase reporter plasmid of pik3r2.

For luciferase assay, the $293 \mathrm{~T}$ cells were seeded into 24-well plates and were co-transfected with miR-126 or $\mathrm{NC}$, and wild-type or mutant-type pik3r2 plasmid. Following incubation for $48 \mathrm{~h}$, the luciferase activity was measured by dual-luciferase assays (E2920; Promega, Madison, WI, USA).

Immunohistochemistry and western blotting. The paraffin-embedded tissues were assessed by immunohistochemistry, or the proteins of cells were extracted using radioimmunoprecipitation buffer for western blotting (Beyotime Institute of Biotechnology, Haimen, China). The detailed process of these two protocols was described previously (20). Protein lysates (30 $\mu \mathrm{g})$ were loaded into $10 \%$ SDS-PAGE gels (Thermo Fisher Scientific, Inc.) and run for $20 \mathrm{~min}$ at 80 and then $120 \mathrm{~V}$, and then transferred to polyvinylidene fluoride membranes (EMD Millipore, Billerica, MA, USA). Following blocking with nonfat milk powder, the membranes were incubated with primary antibodies overnight, followed by $1 \mathrm{~h}$ incubation with the secondary antibody and then were subjected to a chemiluminescence kit (EZ-ECL kit; Biological Industries, Beit Haemek, Israel) For immunohistochemistry, $4 \mu \mathrm{m}$ sections underwent for antigen retrieval by microwave for $25 \mathrm{~min}$ following deparaffinization and hydration. The sections were blocked by $5 \%$ FBS for $1 \mathrm{~h}$ prior to incubation with primary antibody overnight. The sections were then washed with phosphate-buffered saline (Thermo Fisher Scientific, Inc.) and incubated with secondary antibodies prior to treatment with 3,3'-diaminobenzidine staining (ZSGB-BIO, Beijing, China). The primary antibodies used in the present study were as follows: Rabbit polyclonal anti-pik3r2 (1:500; cat. no. ab131067; Abcam, Cambridge, UK), rabbit monoclonal anti-phosphorylated (p-)AKT (1:2,000; cat. no. 2118-1; Epitomics, Burlingame, CA, USA), and rabbit monoclonal anti-GAPDH (1:5,000; cat. no. 5632-1; Epitomics). The secondary antibodies were horseradish peroxidase (HRP)-conjugated goat anti-mouse IgG (heavy + light; 1:3,000; cat. no. SA00001-1; Guangzhou Biological Technology, Co., Ltd., Guangzhou, China) and HRP-conjugated goat anti-rabbit 
A

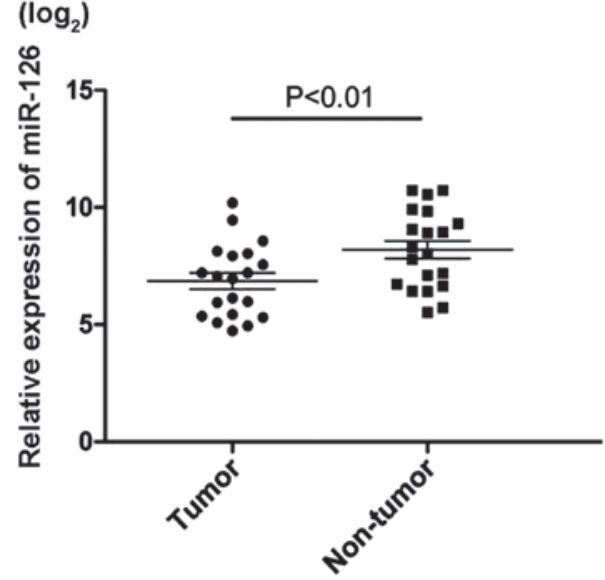

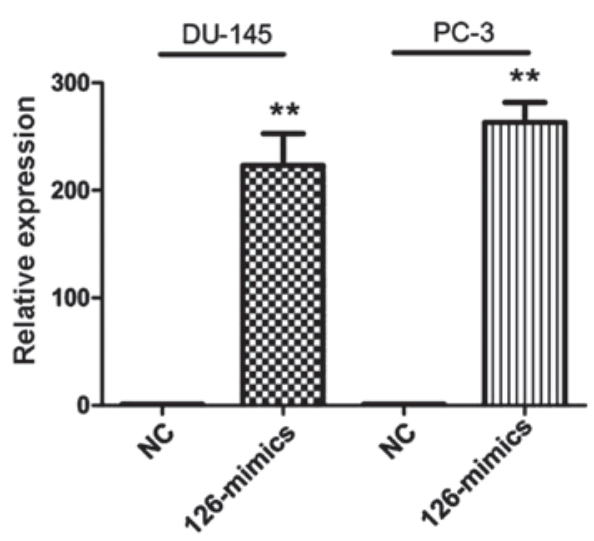

Figure 1. Expression of miR-126 in PCa tissues and miR-126 mimics efficiency in PCa tissues. (A) Reverse transcription-quantitative polymerase chain reaction revealed that the expression of miR-126 was significantly reduced in PCa tissues compared with their normal tissues $(\mathrm{P}<0.01)$. (B) The expression of miR-126 was significantly increased following transfection with the 126 -mimics in two PCa cell lines $\left({ }^{* *} \mathrm{P}<0.01\right)$. miR, microRNA; NC, normal control; PCa, prostate cancer.

A

DU-145 CCK8 assay

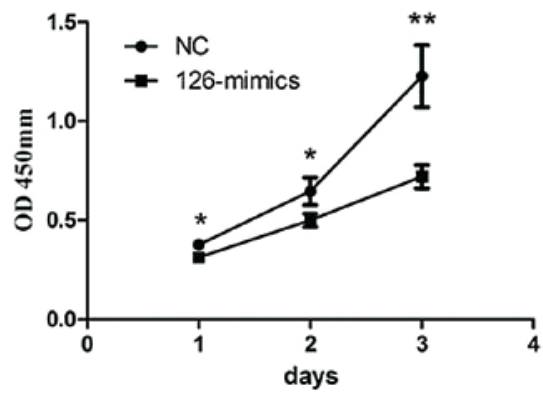

\section{PC-3 CCK8 assay}

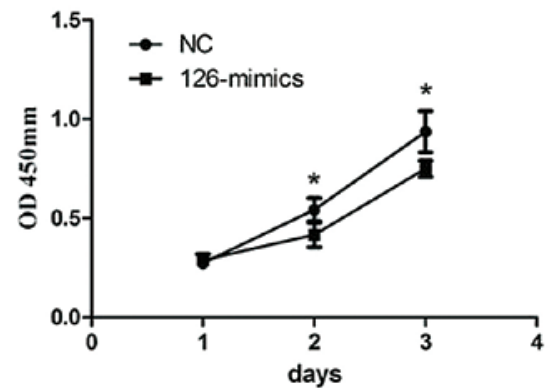

B Stably transfection efficiency

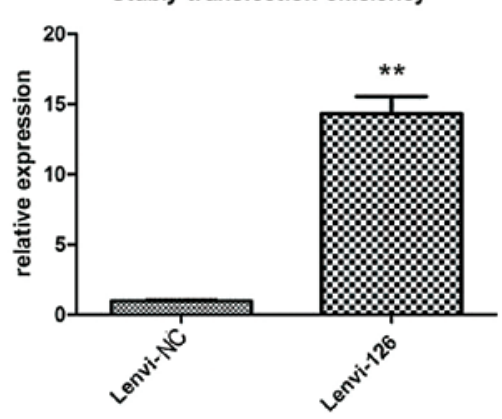

C
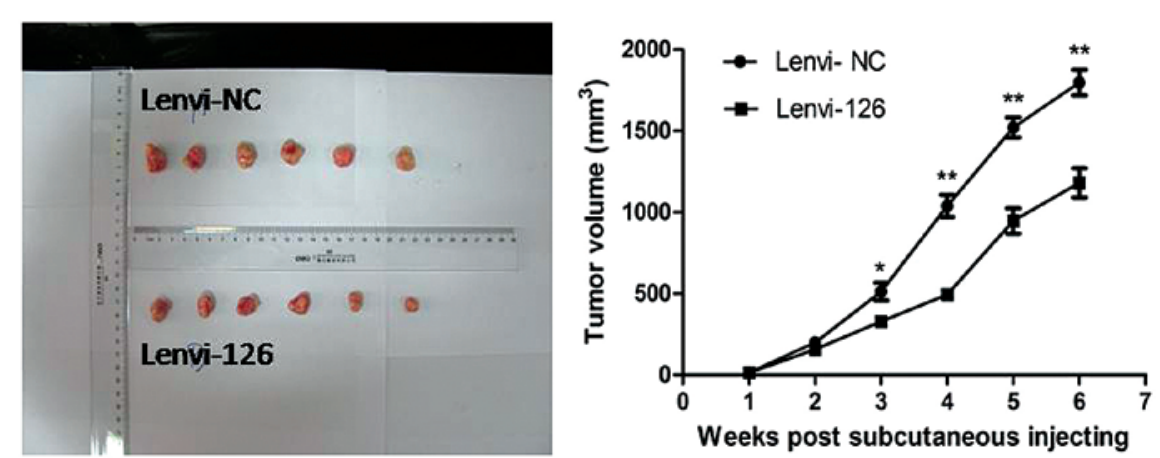

Figure 2. Effects of miR-126 on the proliferation and metastasis of PCa in vitro and in vivo. (A) A CCK-8 assay revealed that miR-126 overexpression suppressed the growth rate of PCa cells. (B) The expression level of miR-126 in stably transfected PC-3 cells was confirmed by reverse transcription-quantitative polymerase chain reaction. (C) The nude mice xenograft model assay revealed that the subcutaneous tumor of the miR-126 stably transfected group proliferated at a markedly slower rate ( ${ }^{*} \mathrm{P}<0.05$ and $\left.{ }^{* *} \mathrm{P}<0.01\right)$. PCa, prostate cancer; CCK, cell counting kit; miR, microRNA; OD, optical density; Lenvi, lentivirus; $\mathrm{nc}$, negative control. 
A
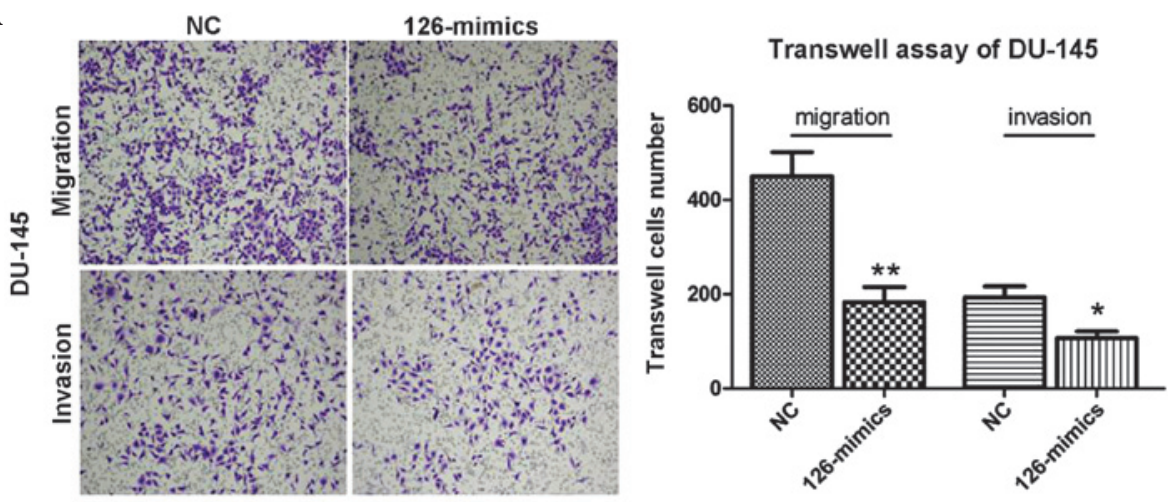

B
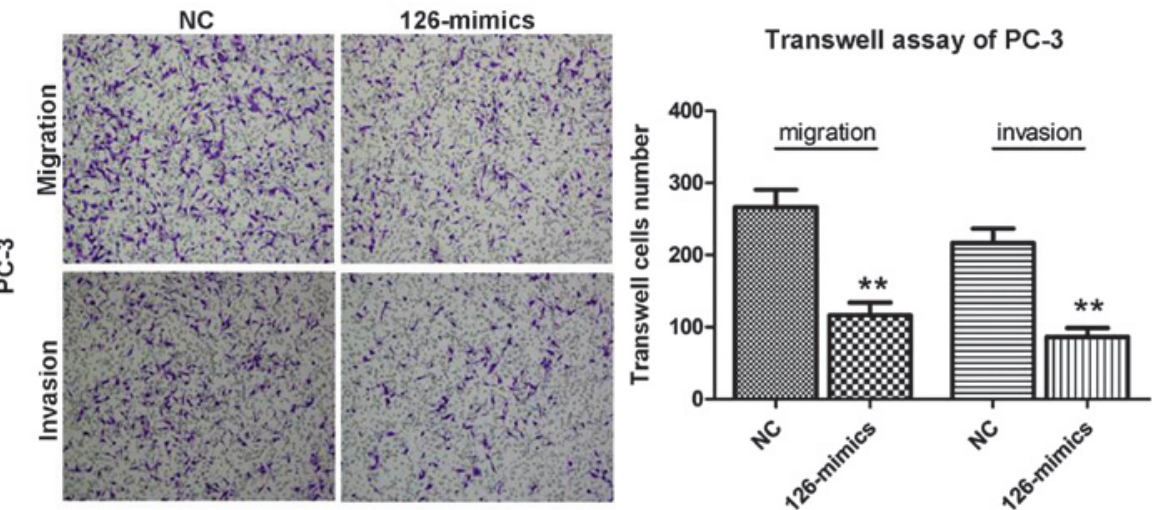

Figure 3. Transwell assays of PCa cells transfected with miR-126 mimics or NC. (A and B) The Transwell assays revealed that the overexpression of miR-126 inhibited the migration and invasion in DU-145 and PC-3 cells. Representative images are shown ( $\mathrm{P}<0.05$ and $\left.{ }^{* *} \mathrm{P}<0.01\right)$. $\mathrm{PC}$, prostate cancer; miR, microRNA; NC, negative control.

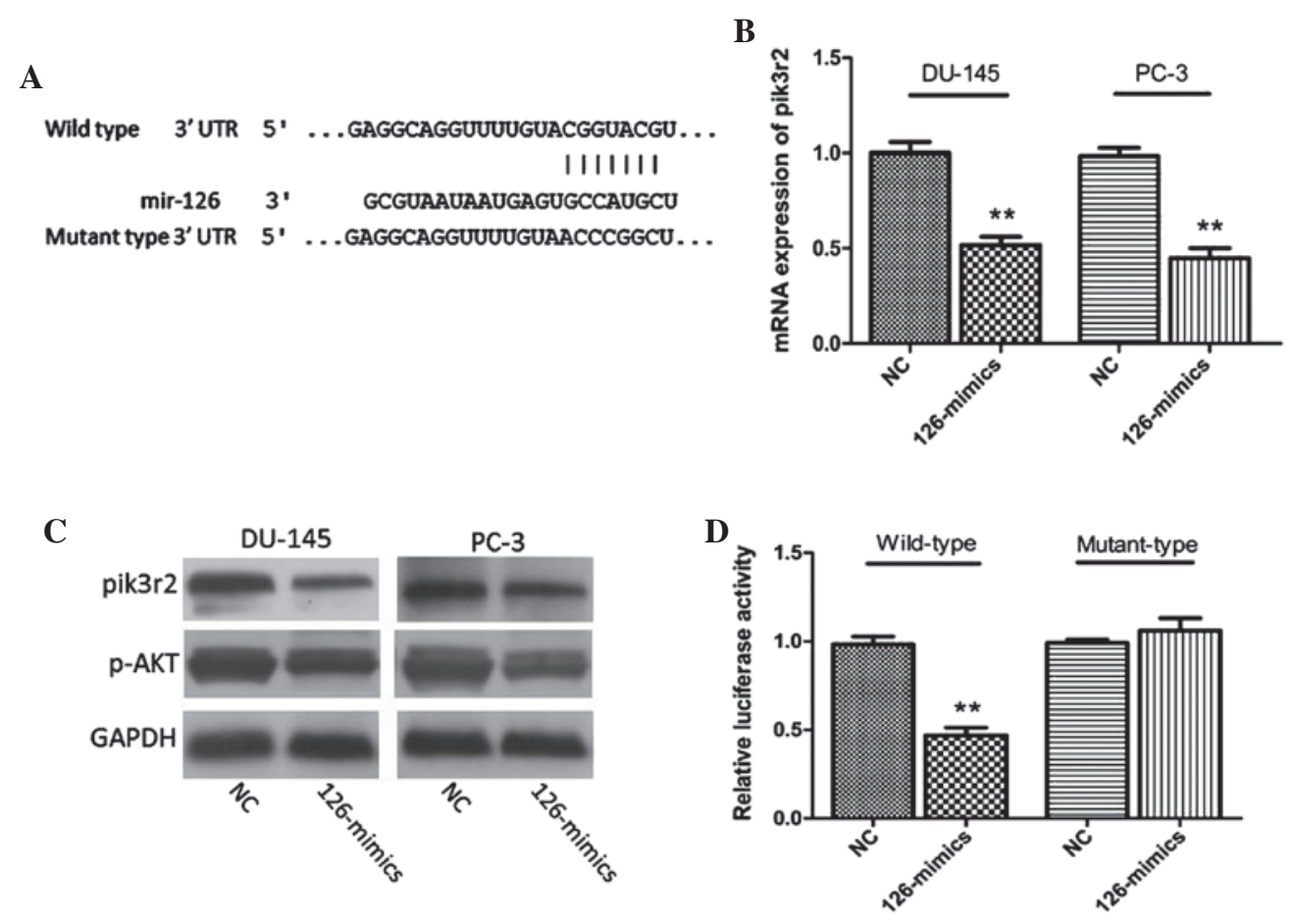

Figure 4. Pik3r2 is a direct target of miR-126 in PCa. (A) Bioinformatics analysis using TargetScan and MiRanda indicated that the 3'UTR of pik3r2 mRNA contains a complementary site for the seed region of miR-126. (B) Reverse transcription-quantitative polymerase chain reaction confirmed that the mRNA expression of pik3r2 was reduced following the overexpression of miR-126 in PCa cells. (C) The western blot assay demonstrated that the protein expression levels of pik3r2 and p-AKT were reduced following overexpression of miR-126 in PCa cells. GAPDH was used as an internal control. (D) The luciferase activity was notably decreased following cotransfected with miR-126 and wt-pik3r2 luciferase plasmid, compared with mt-pik3r2 luciferase plasmid. The results are representative of three independent experiments ( $\left.{ }^{* *} \mathrm{P}<0.01\right)$. miR, microRNA; PCa, prostate cancer; UTR, untranslated region; p-, phosphorylated; wt, wild-type; mt, mutant; NC, negative control. 

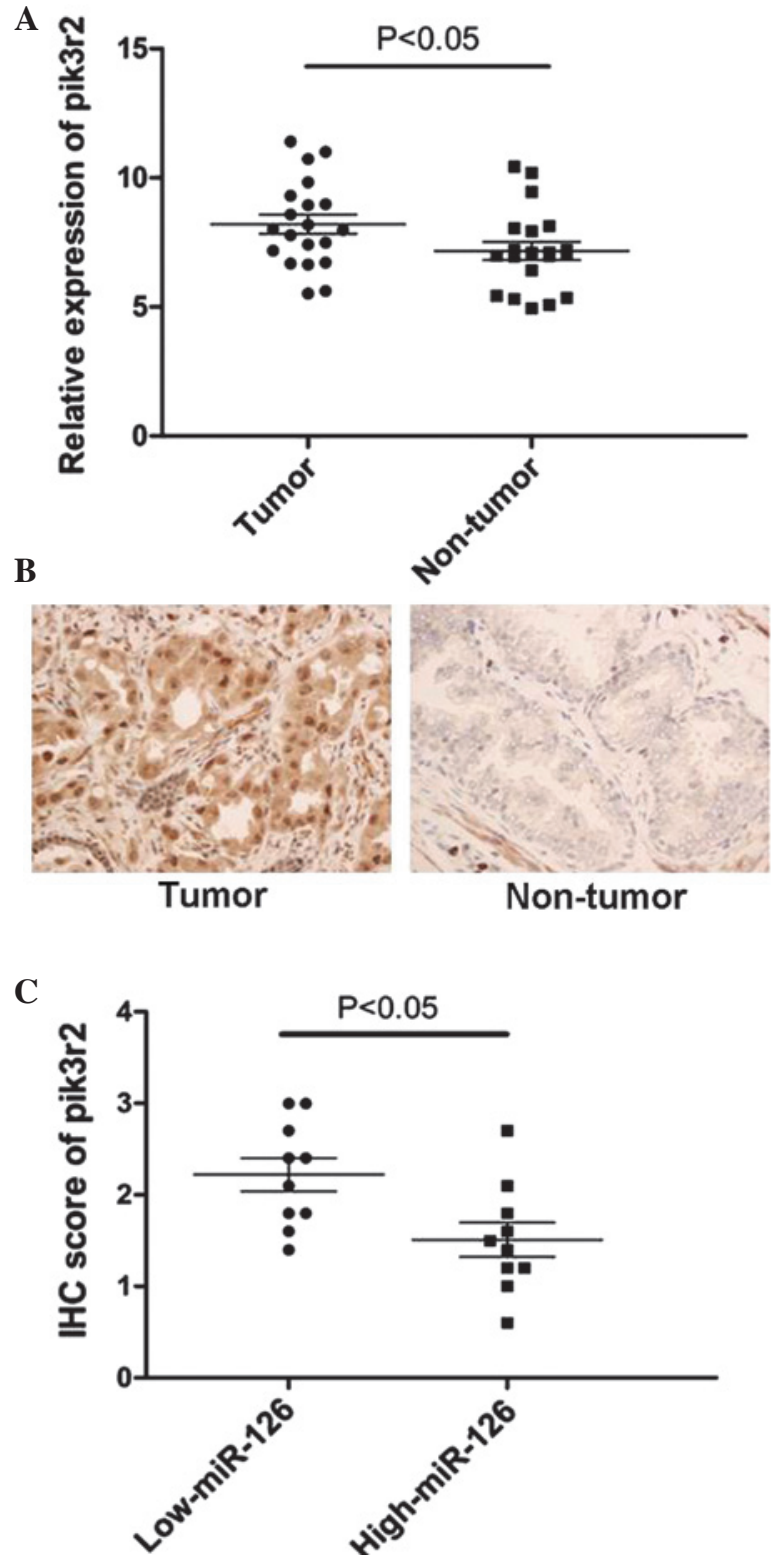

Figure 5. Expression of pik3r2 in $\mathrm{PCa}$ tissues and its correlation with miR-126 in PCa. (A) Reverse transcription-quantitative polymerase chain reaction revealed an increased mRNA expression of pik3r2 in the PCa tissues compared with the normal tissues $(\mathrm{P}<0.05)$. (B) The protein expression of pik3r2 in PCa was detected by immunohistochemistry. Tumor tissues exhibited a higher expression level of pik3r2 compared with the normal tissues. Representative images are shown (magnification, $\mathrm{x} 400$ ). (C) The median of the expression of miR-126 of all 20 PCa tissues was used as the cut-off for separating high-miR-126 $(\mathrm{n}=10)$ from low-miR-126 $(\mathrm{n}=10)$ expressing tumor samples. PCa tumors with lower expression of miR-126 exhibited higher pik3r2 expression $(\mathrm{P}<0.05)$. $\mathrm{PCa}$, prostate cancer; miR, microRNA; IHC immunohistochemical.

IgG (heavy + light; 1:3,000; cat. no. SA00001-2; Guangzhou Biological Technology, Co., Ltd.)

The results of the immunohistochemistry were scored in a semi-quantitative manner. The final score was a composite score obtained by multiplying the values of staining intensity and the percentage of positive cells. Briefly, the staining intensity was categorized as follows: 0 , negative; 1 , weak; 2 , moderate; 3 , strong. The percentage of positive cells was recorded as $0-100 \%$ and the final score, representing the level of pik3r2, ranged between 0 and 3 .
Stable overexpression of miR-126 in PC-3 cells and nude mice xenograft assay. The oligonucleotide sequence of miR-126 (5'-UCGUACCGUGAGUAAUAAUGCG-3') was synthesized, amplified and cloned into a lentiviral vector. For lentiviral infection, PC-3 cells without transfection were eliminated by consistent puromycin incubation and the overexpression of miR-126 in puromycin-resistant PC-3 cells was verified by $\mathrm{RT}-\mathrm{qPCR}$.

Female BALB/c athymic mice (Shanghai Laboratory Animal Research Centre, Shanghai, China; age, 4-6 weeks; weight, 15-18 g) were used and raised under standard animal care conditions with five rats per cage and free access to food and water under a 16-h/8-h light/dark cycle. All animal experiments were performed in accordance with the National Institute of Health Guide for the Care and Use of Laboratory Animals, and were approved by the Ethics Committee of The Second Xiangya Hospital of Central South University. PC-3 cells $\left(1 \times 10^{6}\right)$, stably overexpressing miR-126 or $\mathrm{NC}$, were suspended in $100 \mu \mathrm{l}$ phosphate-buffered saline and were subsequently injected subcutaneously into the posterior flank of the mice. Tumor volumes in the mice were measured with a slide caliper weekly, until the mice were sacrificed at the $6^{\text {th }}$ week. A total of 12 mice were included in the total. The results are presented as the mean \pm standard deviation.

Statistical analysis. Each value in the present study was obtained from at least three independent experiments and the data are expressed as the mean \pm standard deviation. Comparisons between two groups were analyzed by Student's t-test. Statistical analysis was performed using SPSS 15.0 software (SPSS, Inc., Chicago, IL, USA) and GraphPad Prism 5.0 software (GraphPad Software, Inc., La Jolla, CA, USA. $\mathrm{P}<0.05$ was considered to indicate a statistically significant difference.

\section{Results}

miR-126 is significantly downregulated in PCa tissues. To assess the expression of miR-126 in PCa, RT-qPCR was performed in 20 pairs of $\mathrm{PCa}$ tissue. The results showed that $18 \mathrm{PCa}$ tissue samples revealed a lower expression level of miR-126 compared with their corresponding normal tissues and a significant difference was observed between the two groups (Fig. 1A). This suggested that miR-126 may exert an important role in the progression of PCa.

Enhanced expression of miR-126 significantly suppresses the proliferation of $\mathrm{PCa}$ in vitro and in vivo. To assess the potential role of miR-126 in PCa, miR-126 mimics were used to overexpress the level of miR-126 in DU-145 and PC-3 cells (Fig. 1B). A CCK-8 assay was subsequently used to evaluate the proliferation effects of miR-126 in PCa cells. As shown in Fig. 2A, overexpression of miR-126 significantly inhibited the growth rate in the $\mathrm{PCa}$ cell lines.

To further confirm the antiproliferative role of miR-126 in $\mathrm{PCa}$, in vivo proliferation assays were preformed using a nude mice xenograft model. miR-126 or NC were stably transfected into PC-3 cells (Fig. 2B). Following subcutaneous injection for 6 weeks, the tumor volume of the lenvi-126 group revealed a slower proliferation trend compared with the lenvi-NC 
group (Fig. 2C). The data suggested that overexpression of miR-126 significantly inhibited the proliferation in vitro and in vivo.

miR-126 inhibits metastasis in DU-145 and PC-3 cells. Transwell assays were performed to examine the metastasis potential of miR-126. As shown in Fig. 3, the invasion and migration activities were markedly reduced following the overexpression of miR-126 in DU-145 and PC-3 cells. The results indicated the suppressive role of miR-126 in metastasis in PCa cells.

Pik3r2 is a direct target of miR-126 in PCa cells and tissues. As the functions of miRNAs are mediated by regulating target genes (21), the present study sought to identify a potential target of miR-126 in PCa. By comprehensive analysis of the bioinformatics websites, TargertScan and miRanda, the gene, pik3r2, was identified (Fig. 4A). This gene is a well-known component of the PI3K/p-AKT pathway (22). As shown in Fig. 4B and $\mathrm{C}$, the mRNA and protein expression levels of pik3r2 in DU-145 and PC-3 cells were markedly decreased following treatment with miR-126 mimics. In addition, western blotting indicated that the protein expression of p-AKT was also reduced, accompanied by the decrease in pik3r2 (Fig. 4C). A luciferase reporter assay was subsequently performed in 293T cells to further confirm the direct interaction of miR-126 and pik3r2. MiR-126 overexpression decreased the luciferase activity of the wild type pik3r2 plasmid, while there were no effects of miR-126 mimics when the binding sites of pik3r2 plasmid were mutated (Fig. 4D).

Based on the observation that the expression level of miR-126 was markedly downregulated in 20 PCa tissues, RT-qPCR and immunohistochemistry assays of pik3r2 were performed in these 20 tissue samples. By contrast, the expression of pik3r2 was significantly upregulated in PCa tissues compared with their corresponding normal tissues (Fig. 5A and $\mathrm{B}$ ). These PCa tissues were subsequently divided into two groups by selecting the median of miR-126 expression of all $20 \mathrm{PCa}$ tissues as the cut-off. It was then revealed that the expression of miR-126 inversely correlated with the expression of pik3r2 in these $20 \mathrm{PCa}$ tissues (Fig. 5C). Taken together, the results demonstrated that pik3r2 is a direct target of miR-126 in vitro and in vivo in PCa.

\section{Discussion}

$\mathrm{PCa}$ is one of most frequent and malignant types of cancer in men, which owns an increasing morbidity and mortality rate. Over past decades, researchers have investigated the underlying molecular mechanism involved in the initiation and progression of PCa. miRNAs are hypothesized to be a highly involved in mediating malignant biological functions in cancer development $(23,24)$. In PCa, several miRNAs are demonstrated to have a vital role in the process of antiapoptosis (25), pro-proliferation (26) and pro-metastasis (27).

To date, the role of miR-126 in PCa, particularly its functional role, remains to be elucidated. In the present study, it was demonstrated that the expression of miR-126 was significantly lower in the 20 PCa cases compared with their corresponding normal tissues, which is consistent with a previous report (19). It was subsequently demonstrated that overexpression of miR-126 suppressed the proliferation and inhibited the metastasis in two PCa cell lines, DU-145 and PC-3. Using nude mice xenograft models, it was also revealed that miR-126 markedly suppressed the growth of subcutaneous tumors. Additionally, it was confirmed that pik3r2 was a direct target gene of miR-126 in vitro and in vivo. This is the first attempt, to the best of our knowledge, to illuminate the role of miR-126 deregulation in the proliferation and metastasis of $\mathrm{PCa}$, using both in vitro and in vivo models.

A high frequency of proliferation is a basic characteristic of cancer, and metastasis is the major cause of cancer mortality, while angiogenesis is essential for tumor growth and metastasis (28). Therefore, the identification of novel therapeutics targeting proliferation and metastasis may be beneficial for clinical applications. miR-126 has been shown to affect numerous cellular processes, particularly inhibiting angiogenesis and metastasis in cancer $(29,30)$, which suggests that it is important in cancer progression. However, its potential role in $\mathrm{PCa}$ remains to be elucidated. The present data revealed a decreased expression of miR-126 in $\mathrm{PCa}$ tissues, suggesting a potential tumor suppressor role in $\mathrm{PCa}$. Subsequent functional assays revealed the antiproliferative and antimetastatic effects of miR-126 in PCa cells, which were in agreement with the role of miR-126 in other cancer types.

$\mathrm{Pik} 3 \mathrm{r} 2$ is a regulatory component of $\mathrm{PI} 3 \mathrm{~K}$, which is located on the upstream of AKT (31). The PI3K/p-AKT pathway is well-known to be responsible for regulating cell growth, proliferation, survival and angiogenesis in the development of cancer. Therefore, molecules altering the expression of pik3r2 may be an effective way to lower the malignancy of cancer types. Several previous reports have demonstrated that miR-126 reduces the expression of pik3r2 by directly targeting its 3 '-untranslated region $(32,33)$. Similarly, the present data revealed that pik3r2 is a target gene of miR-126 in PCa, based on the following evidence: i) The mRNA and protein expression levels of pik3r2 were significantly decreased following the overexpression of miR-126 in PCa cells; ii) The expression levels of miR-126 and pik3r2 were inversely correlated in PCa tissues. In the present study, it was also demonstrated that the expression of p-AKT was markedly decreased, accompanied by downregulation of pik3r2 following the overexpression of miR-126. Since the activation of $\mathrm{p}-\mathrm{AKT}$ is an important mechanism to maintain proliferation and metastasis in the initiation and progression of cancer development $(34,35)$, it was concluded that the biological mechanism of miR-126 in $\mathrm{PCa}$ is potentially mediated by the PI3K/p-AKT pathway.

In conclusion, the present study suggested that downregulation of miR-126 is a frequent event in $\mathrm{PCa}$ and restoration of miR-126 significantly inhibited the proliferation and metastasis of PCa via the PI3K/p-AKT pathway. Therefore, miR-126 may be a potential target for PCa therapy.

\section{Acknowledgements}

The authors would like to thank all doctors and staff who contributed to the sample recruitment and information collection. 


\section{References}

1. Yamamoto S, Kawakami S, Yonese J, Fujii Y, Urakami S, Masuda H, Numao N, Ishikawa Y, Kohno A and Fukui I: Long-term oncological outcome and risk stratification in men with high-risk prostate cancer treated with radical prostatectomy. Jpn J Clin Oncol 42: 541-547, 2012.

2. Peyromaure EM, Mao K, Sun Y, Xia S, Jiang N, Zhang S, Wang G, Liu Z and Debré B: A comparative study of prostate cancer detection and management in China and in France. Can J Urol 16: 4472-4477, 2009.

3. Klotz L: Active surveillance for prostate cancer: For whom? J Clin Oncol 23: 8165-8169, 2005

4. Walz J, Gallina A, Saad F, Montorsi F, Perrotte P, Shariat SF Jeldres C, Graefen M, Bénard F, McCormack M, et al: A nomogram predicting 10-year life expectancy in candidates for radical prostatectomy or radiotherapy for prostate cancer. J Clin Oncol 25: 3576-3581, 2007.

5. Siegel R, Ward E, Brawley O and Jemal A: Cancer statistics, 2011: The impact of eliminating socioeconomic and racial disparities on premature cancer deaths. CA Cancer J Clin 61: 212-236, 2011.

6. Vaiopoulos AG, Athanasoula KCh and Papavassiliou AG: Epigenetic modifications in colorectal cancer: Molecular insights and therapeutic challenges. Biochim Biophys Acta 1842: 971-980, 2014.

7. Romero Otero J, Garcia Gomez B, Campos Juanatey F and Touijer KA: Prostate cancer biomarkers: An update. Urol Oncol 32: 252-260 2014

8. Lee RC, Feinbaum RL and Ambros V: The C.elegans heterochronic gene lin-4 encodes small RNAs with antisense complementarity to lin-14. Cell 75: 843-854, 1993.

9. Calin GA and Croce CM: MicroRNA signatures in human cancers. Nat Rev Cancer 6: 857-866, 2006.

10. Petri A, Lindow $M$ and Kauppinen S: MicroRNA silencing in primates: Towards development of novel therapeutics. Cancer Res 69: 393-395, 2009.

11. Yang J, Zhao H, Xin Y and Fan L: MicroRNA-198 inhibits proliferation and induces apoptosis of lung cancer cells via targeting FGFR1. J Cell Biochem 115: 987-995, 2014.

12. Nassirpour R, Mehta PP and Yin MJ: miR-122 regulates tumorigenesis in hepatocellular carcinoma by targeting AKT3. PLoS One 8: e79655, 2013

13. Ouyang H, Gore J, Deitz S and Korc M: microRNA-10b enhances pancreatic cancer cell invasion by suppressing TIP30 expression and promoting EGF and TGF- $\beta$ actions. Oncogene 33: 4664-4674, 2014.

14. Fang JH, Zhou HC, Zeng C, Yang J, Liu Y, Huang X, Zhang JP, Guan XY and Zhuang SM: MicroRNA-29b suppresses tumor angiogenesis, invasion and metastasis by regulating matrix metalloproteinase 2 expression. Hepatology 54: 1729-1740, 2011.

15. Liu D, Tao T, Xu B, Chen S, Liu C, Zhang L, Lu K, Huang Y, Jiang L, Zhang X, et al: MiR-361-5p acts as a tumor suppressor in prostate cancer by targeting signal transducer and activator of transcription-6 (STAT6). Biochem Biophys Res Commun 445: 151-156, 2014.

16. Fu X, Meng Z, Liang W, Tian Y, Wang X, Han W, Lou G, Wang X, Lou $\mathrm{F}$, Yen Y, et al: miR-26a enhances miRNA biogenesis by targeting Lin28B and Zcchc11 to suppress tumor growth and metastasis. Oncogene 33: 4296-4206, 2014.

17. Huang TH and Chu TY: Repression of miR-126 and upregulation of adrenomedullin in the stromal endothelium by cancer-stromal cross talks confers angiogenesis of cervical cancer. Oncogene 33: 3636-3647, 2014.

18. Li Z, Li N, Wu M, Li X, Luo Z and Wang X: Expression of miR-126 suppresses migration and invasion of colon cancer cells by targeting CXCR4. Mol Cell Biochem 381: 233-242, 2013.
19. Sun X, Liu Z, Yang Z, Xiao L, Wang F, He Y, Su P, Wang J and Jing B: Association of microRNA-126 expression with clinicopathological features and the risk of biochemical recurrence in prostate cancer patients undergoing radical prostatectomy. Diagn Pathol 8: 208, 2013.

20. Cheng J, Xie HY, Xu X, Wu J, Wei X, Su R, Zhang W, Lv Z, Zheng $S$ and Zhou L: NDRG1 as a biomarker for metastasis, recurrence and of poor prognosis in hepatocellular carcinoma. Cancer Lett 310: 35-45, 2011.

21. Bartel DP: MicroRNAs: Genomics, biogenesis, mechanism and function. Cell 116: 281-297, 2004.

22. Bagchi P, Nandi S, Nayak MK and Chawla-Sarkar M: Molecular mechanism behind rotavirus NSP1-mediated PI3 kinase activation: Interaction between NSP1 and the p85 subunit of PI3 kinase. J Virol 87: 2358-2362, 2013.

23. Mulrane L, McGee SF, Gallagher WM and O'Connor DP: miRNA dysregulation in breast cancer. Cancer Res 73: 6554-6562, 2013.

24. Cai J, Yang C, Yang Q, Ding H, Jia J, Guo J, Wang J and Wang Z: Deregulation of let-7e in epithelial ovarian cancer promotes the development of resistance to cisplatin. Oncogenesis 2: e75, 2013.

25. Li X, Chen YT, Josson S, Mukhopadhyay NK, Kim J, Freeman MR and Huang WC: MicroRNA-185 and 342 inhibit tumorigenicity and induce apoptosis through blockade of the SREBP metabolic pathway in prostate cancer cells. PLoS One 8: e70987, 2013

26. Tian L, Fang YX, Xue JL and Chen JZ: Four microRNAs promote prostate cell proliferation with regulation of PTEN and its downstream signals in vitro. PLoS One 8: e75885, 2013.

27. Kao CJ, Martiniez A, Shi XB, Yang J, Evans CP, Dobi A, deVere White RW and Kung HJ: miR-30 as a tumor suppressor connects EGF/Src signal to ERG and EMT. Oncogene 33: 2495-2503, 2014.

28. Kerbel RS: Tumor angiogenesis. N Engl J Med 358: 2039-2049, 2008.

29. Zhou Y, Feng X, Liu YL, Ye SC, Wang H, Tan WK, Tian T, Qiu YM and Luo HS: Down-regulation of miR-126 is associated with colorectal cancer cells proliferation, migration and invasion by targeting IRS-1 via the AKT and ERK1/2 signaling pathways. PLoS One 8: e81203, 2013.

30. de Giorgio A, Castellano L, Krell J and Stebbing J: Crosstalk-induced loss of miR-126 promotes angiogenesis. Oncogene 33: 3634-3635, 2014.

31. Coutte L, Dreyer C, Sablin MP, Faivre S and Raymond E: PI3K-AKT-mTOR pathway and cancer. Bull Cancer 99: 173-180, 2012 (In French).

32. Fish JE, Santoro MM, Morton SU, Yu S, Yeh RF, Wythe JD, Ivey KN, Bruneau BG, Stainier DY and Srivastava D: miR-126 regulates angiogenic signaling and vascular integrity. Dev Cell 15: 272-284, 2008.

33. Zhang J, Zhang Z, Zhang DY, Zhu J, Zhang T and Wang C: microRNA 126 inhibits the transition of endothelial progenitor cells to mesenchymal cells via the PIK3R2-PI3K/Akt signalling pathway. PLoS One 8: e83294, 2013.

34. Skeen JE, Bhaskar PT, Chen CC, Chen WS, Peng XD, Nogueira V, Hahn-Windgassen A, Kiyokawa $\mathrm{H}$ and Hay $\mathrm{N}$ : Akt deficiency impairs normal cell proliferation and suppresses oncogenesis in a p53-independent and mTORC1-dependent manner. Cancer Cell 10: 269-280, 2006

35. Phung TL, Ziv K, Dabydeen D, Eyiah-Mensah G, Riveros M, Perruzzi C, Sun J, Monahan-Earley RA, Shiojima I, Nagy JA, et al: Pathological angiogenesis is induced by sustained Akt signaling and inhibited by rapamycin. Cancer Cell 10: 159-167 2006. 